Three new records in the zone of influence of the

Biosphere Reserve Tehuacan-Cuicatlan, Oaxaca

\title{
Tres nuevos registros en la zona de influencia de la Reserva de la Biosfera Tehuacán- Cuicatlán, Oaxaca
}

\author{
Alejandro Espinosa-Lucas ${ }^{1,2 *}$, Ángel Méndez ${ }^{2}$, Omar Hernández ${ }^{2}$, Adolfo Flores-Cortés², Francisco Botello ${ }^{1,2}$ e \\ Isaac Mariscal ${ }^{3}$
}

\begin{abstract}
1 Departamento de Zoología, Instituto de Biología, Universidad Nacional Autónoma de México. Av. Ciudad Universitaria 3000, Apartado Postal 70-153, Coyoacán 04360. Distrito Federal, México. Email: alejandroespinosa@conbiodes.com (AE-L), franciscobotello@conbiodes.com (FB).

${ }^{2}$ Departamento de monitoreo biológico, Conservación Biológica y Desarrollo Social A. C. Calle Nueve, núm. 52, int. 4. Coyoacán 04870. Distrito Federal, México. Email: angelmendez@conbiodes.com (AM),omarhernandez@conbiodes.com(OH), adolfoflores@ conbiodes.com (AF-C),

3 Red de monitoreo de fauna silvestre, Calle Nueve, núm. 52, int. 4. Coyoacán 04870. Distrito Federal, México. Email: comisariado_19@hotmail.com (IM).

*Corresponding author
\end{abstract}

Introduction: Tamandua mexicana is the only species of anteater present throughout Mexico. Whereas Galictis vittata and Eira barbara are two species of endangered mustelids according to the mexican's laws. We present records for the three species mentioned, located within the area of influence of the Biosphere Reserve Tehuacan-Cuicatlan (RBTC), in the state of Oaxaca.

Methodology: Over five years, a strong link to various human communities located within the RBTC has consolidadted, conforming to date 19 participatory monitoring groups, each represented by a different municipality. Each of the groups are familiar with the use of camera traps, and their correct location in the field.

Results: The noteworthy records of Tamandua mexicana, Galictis vittata and Eira barbara for the zone of influence of the RBTC were obtained. The record of T. mexicana was obtained in the town of Concepcion Papalo in pine-oak forest. The records of mustelids were obtained in the town of Santa Maria Papalo, in cloud forest.

Discussion and conclusions: These records obtained for the area of influence of the RBTC provides new information for the three species and increases the list of mammals adjacent to the reserve. The record of the anteater increases its altitudinal range reported approximately $500 \mathrm{~m}$. Whereas for mustelids our information is relevant because of their critical situation and the limited information which has been marked as priority for the Mustelidae Family.

Key words: Altitude; camera trapping; forest; participatory monitoring; record; small carnivores.

Introducción: Tamandua mexicana es la única especie de oso hormiguero presente en México. En cambio, de acuerdo con las leyes mexicanas, Galictis vittata y Eira barbara son dos especies de mustélidos en peligro de extinción. En este trabajo, presentamos los primeros registros de las tres especies mencionadas, que se ubican en la zona de influencia de la Reserva de la Biosfera Tehuacán-Cuicatlán (RBTC), en el estado de Oaxaca.

Metodología: A lo largo de cinco años, Conbiodes A. C., ha consolidado una fuerte vinculación con diversas comunidades humanas en la RBTC. A la fecha, conforman 19 grupos de monitoreo participativo. Cada uno representa a un municipio diferente y se halla familiarizado con el uso de fototrampas y con su correcta ubicación en el campo.

Resultados: Se obtuvo registros notables de Tamandua mexicana, Galictis vittata y Eira barbara en la zona de influencia de la RBTC. El registro de T. mexicana se obtuvo en el municipio de Concepción Pápalo, en bosque de pino-encino. Los registros de mustélidos fueron obtenidos en el municipio de Santa María Pápalo, en bosque mesófilo de montaña.

Discusión y conclusiones: Los registros obtenidos en la zona de influencia de la RBTC aportan nueva información 
sobre las tres especies y aumentan la lista de mamíferos aledaños a la reserva. El registro de oso hormiguero aumenta cerca de $500 \mathrm{~m}$ su intervalo altitudinal reportado. Se trata de información relevante sobre los mustélidos, debido a la situación crítica en que se encuentran y a la escasa información, que ha sido marcada como prioritaria para la Familia Mustelidae.

Palabras clave: Altitud; bosque; carnívoros pequeños; fototrampeo; monitoreo participativo; registro.

\section{Introducción}

Oaxaca se encuentra entre los estados de México más importantes en términos de diversidad biológica. En el ámbito nacional, ocupa el tercer lugar de refugio de artrópodos y plantas vasculares, con 4,204 y 4,085 especies respectivamente (Llorente-Bousquets et al. 2008). En el caso de vertebrados, ocupa el segundo lugar, con un total de 1,322 especies (Llorente-Bousquets y Ocegueda 2008). Dentro de su territorio, compartido con el estado de Puebla, se ubica una de las áreas naturales protegidas más extensas y diversas de México (Dávila et al. 2002; Ramírez-Pulido y Martínez-Vázquez 2007; SEMARNAT 2010), la Reserva de la Biosfera Tehuacán-Cuicatlán (RBTC).

Actualmente, el fototrampeo ha beneficiado mucho el conocimiento mastozoológico, ya que se trata de una técnica no invasiva e ideal para documentar la fauna difícil de detectar por su baja probabilidad de captura, ya sea por su comportamiento críptico o por sus bajas densidades ( $\mathrm{O}^{\prime}$ Connell et al. 2011). En la RBTC, gracias a esta técnica, se ha logrado documentar, por primera vez, la presencia de varias especies (Botello et al. 2013). Por lo anterior, el objetivo de este trabajo fue incrementar el conocimiento mastofaunístico y documentar los registros de Eira barbara, Galictis vittata y Tamandua mexicana, ubicados en la zona de influencia

\section{Material y métodos}

Geográficamente, la poligonal de la RBTC se ubica, en su extremo norte, en las coordenadas $18.876781^{\circ} \mathrm{N}$ y $-97.692047^{\circ} \mathrm{O}$; en su extremo sur, en las coordenadas $17.542497^{\circ} \mathrm{N}$ y $-96.720472^{\circ}$ $\mathrm{O}$, y en sus extremos este y oeste, en las coordenadas $17.649167^{\circ} \mathrm{N}$ y $-96.692047^{\circ} \mathrm{O}$ y $-97.809792^{\circ}$ O, respectivamente. La superficie total de la Reserva es de aproximadamente 490 mil hectáreas (SEMARNAT 2013). Sus principales tipos de vegetación son la selva baja caducifolia, matorral xerófilo, bosques de encino, bosque de pino-encino y bosque mesófilo de montaña. La temperatura ambiental oscila entro los $12^{\circ} \mathrm{C}$ y los $24^{\circ} \mathrm{C}$ (SEMARNAT 2013).

La zona de influencia está conformada por 51 municipios que mantienen una porción territorial dentro del polígono decretado para la Reserva. La ubicación geográfica de la zona de influencia se localiza entre las coordenadas limitantes al norte, $18.965278^{\circ} \mathrm{N}$ y $-97.533417^{\circ} \mathrm{O}$; al sur, $17.433389^{\circ}$ $\mathrm{Ny}-96.883356^{\circ} \mathrm{O}$, y los límites de las coordenadas longitudinales se encuentra en los $18.466828^{\circ}$ $\mathrm{Ny}-96.850117^{\circ} \mathrm{O}$ al este, y a $18.100025^{\circ} \mathrm{N}$ y $-97.850106^{\circ} \mathrm{O}$ al oeste. Su área es de 324,045 ha, que rodean el polígono de la RBTC (SEMARNAT 2013).

Desde su inicio en 2010, en la RBTC se ha consolidado una red de monitoreo participativo coordinada por Conservación Biológica y Desarrollo Social A. C. (Conbiodes), mediante el método de fototrampeo. Ha estado vigente hasta la fecha. Con el respaldo de conocimiento local y científico, se han logrado registrar especies que han permitido incrementar el conocimiento de la zona (Botello et al. 2013). Actualmente, se cuenta con la participación de 19 núcleos agrarios involucrados en el trabajo, los cuales utilizan la técnica de fototrampeo. Cada núcleo cuenta, en promedio, con cuatro fototrampas Ltl Acorn, 12 megapixeles, lo que da un total de 108 fototrampas. Cada una está instalada aproximadamente a $40 \mathrm{~cm}$ del piso, con una separación de $400 \mathrm{~m}$ lineales entre cada estación. Cada cámara permanece activa por un periodo aproximado de dos meses. Finalizado este tiempo, a cada fototrampa se le cambian las pilas y la memoria. Las imágenes son sistematizadas. La asignación a nivel de especie se hace con la ayuda de literatura especializada 
(Ceballos y Oliva 2005; Aranda 2012). Finalmente, las imágenes son albergadas en la Colección de Fotocolectas Biológicas del Instituto de Biología de la Universidad Nacional Autónoma de México (IBUNAM).

\section{Resultados}

Con base en el trabajo de esta red de monitoreo participativo, se ha obtenido durante el periodo de 2010 a 2012 un total de 13,697 imágenes. Los resultados que presentamos aquí pertenecen a tres diferentes periodos de muestreo. El primero va del 8 de septiembre al 2 de noviembre de 2012; el segundo, del 7 de mayo al 18 de mayo de 2013, y el tercero, del 31 de mayo al 13 de septiembre de 2013. Estos tres periodos, en conjunto, produjeron la cantidad total de 89 registros. Entre estas imágenes, destacaron registros relevantes de T. mexicana (Figura 1A), G. vittata (Figura 1B) y E. barbara (Figura 1C) en la zona de influencia de la RBTC.

El 9 de mayo de 2013, con un esfuerzo de captura de dos días-trampa, y con un esfuerzo total de 11 días-trampa, se logró captar por única ocasión a T. mexicana (IBUNAM-CFB-17410; Figura $2 \mathrm{~A}$ ) en una vegetación acorde con la serie IV del INEGI (bosque mesófilo de montaña, aunque la vegetación apreciable en campo es de pino-encino). Lo anterior se llevó a cabo en el municipio de Concepción Pápalo, a una elevación de 2,694 m, en las coordenadas Latitud $17.8623^{\circ} \mathrm{N}$ y Longitud $-96.82275^{\circ} \mathrm{O}$.

En cuanto a E. barbara (IBUNAM-CFB-17171; Figura 2B), se obtuvo su único registro con un

Figura 1. Registros notables de: A) Tamandua mexicana ubicado en el municipio de Concepción Pápalo, Oaxaca; B) Eira barbara, y C) Galictis vittata, ambos ubicados en el municipio de Santa María Pápalo, Oaxaca. En cada uno de los mapas, el círculo representa los registros históricos; el cuadrado, al registro notable, y el rectángulo, al polígono de la Reserva.
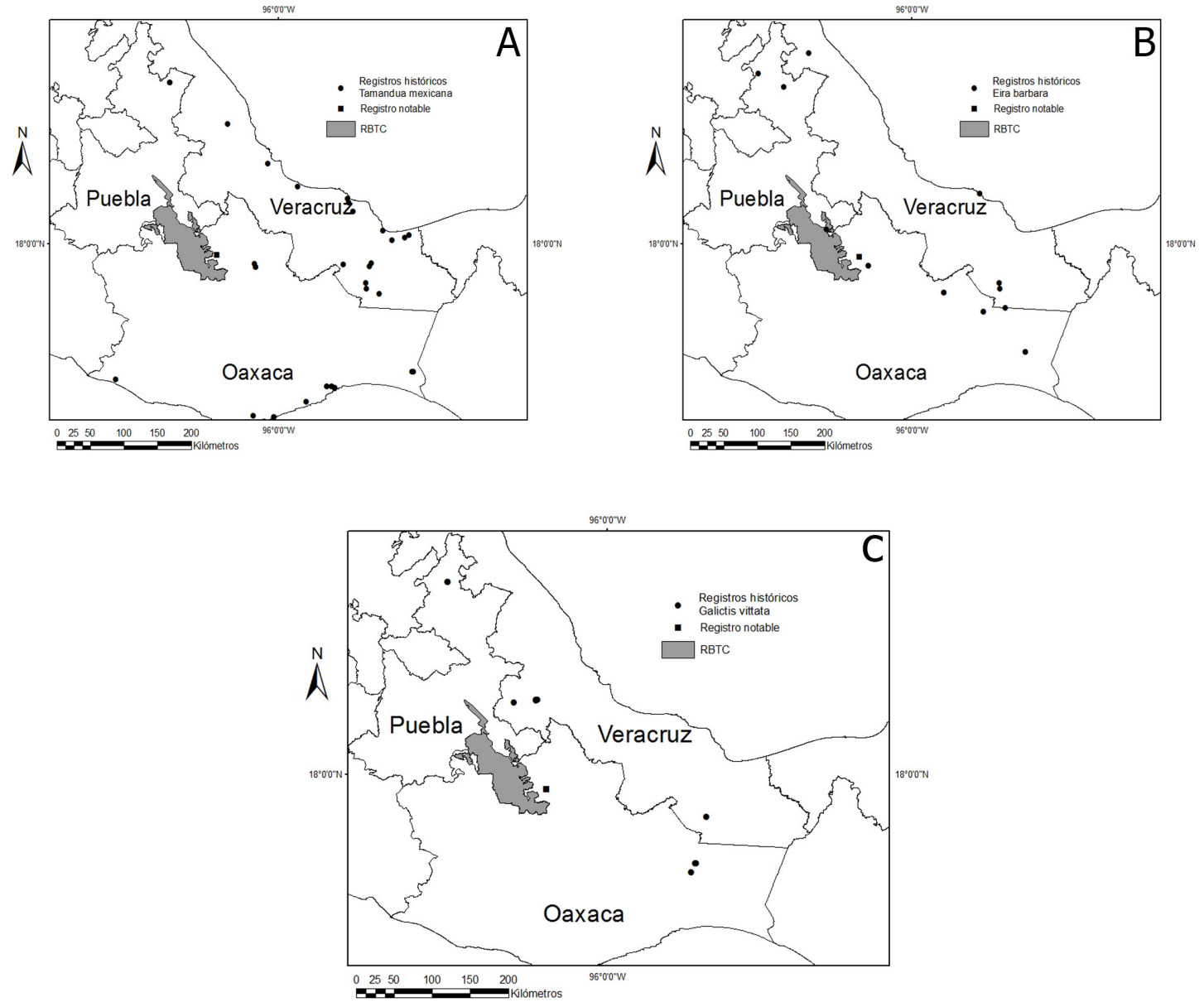
esfuerzo de captura de siete días-trampa de un total de 75 días-trampa, el 31 de octubre de 2012 en las coordenadas $17.8385^{\circ} \mathrm{N}$ y $-96.7067^{\circ} \mathrm{O}$, a una elevación de $921 \mathrm{~m}$. Lo anterior se llevó a cabo en el municipio de Santa María Pápalo, en bosque mesófilo de montaña.

La única imagen de G. vittata (IBUNAM-CFB-17412; Figura 2C) fue captada el 12 de junio de 2013 , en bosque mesófilo de montaña, en las coordenadas $17.83002^{\circ} \mathrm{N}$ y $-96.71861^{\circ} \mathrm{O}$, a una elevación de 1,226 m, con un esfuerzo de captura de 12 días-trampa de un total de 103 díastrampa.

\section{Discusión}

Los registros de las tres especies adquieren relevancia para la zona (Briones-Salas 2000; Dávila et al. 2002; Ramírez-Pulido y Martínez-Vázquez 2007). En el caso de T. mexicana, la elevación máxima reportada era de 2,200 en la municipio de Ángel Albino Corzo, Chiapas, en las coordenadas $15.672^{\circ} \mathrm{N}$ y $-92.82^{\circ} \mathrm{N}$ (GBIF; http://www.gbif.org). Con el registro reportado se incrementa la información de su presencia respecto del intervalo altitudinal y se amplía cerca de $500 \mathrm{~m}$ (Cuarón 2005; Navarrete y Ortega 2011; Núñez-Pérez et al. 2011; GBIF; http://www.gbif.org ).

Los registros de los mustélidos (E. barbara y G. vittata) adquieren importancia por la escasa información que se tiene sobre estas especies en el territorio nacional, y más aún en las zonas aledañas a la Reserva. La información es de gran relevancia, dado el estado crítico en que se encuentran ambas especies, catalogadas en la NOM-059-SEMARNAT-2010 (en peligro de extinción). En cuanto a E. barbara, aunque se reconoce su presencia en Oaxaca (Pérez-Irineo y Santos-Moreno 2012) y cerca de la Reserva por un avistamiento, en el municipio de Coxcatlán de Osorio (RamírezPulido et al. 2005), esta es la primera ocasión que se registra de manera fotográfica en la zona de influencia de la RBTC. Lo anterior aporta información básica de dicha especie, de la que existe poca información en el país (López y Aceves 2007; Ramírez-Bravo 2011). Respecto a G. vittata, su presencia reportada más cercana a la RBTC se ubicaba en el municipio de Orizaba, Veracruz (GBIF; http://www.gbif.org). Con el registro que presentamos, se aumenta el conocimiento de la especie, lo cual es clave, ya que la información del género Galictis es de las más escasas sobre los mustélidos (Bornholdt et al. 2013), y la generación de conocimiento de carnívoros pequeños es una prioridad (Oliveira 2009). En el caso particular de T. mexicana y E. barbara, es importante mencionar que son de hábitos arborícolas (Presley 2000; Navarrete y Ortega 2011); por lo tanto, se requiere formalizar estudios que incluyan el monitoreo del estrato arbóreo en la región.

Con base en lo señalado, es necesario realizar programas de monitoreo a largo plazo, que aseguren el registro de especies con conductas crípticas y que ayuden a generar información sobre estas especies: no sólo sobre su distribución, sino también sobre su ecología, ya que probablemente

Figura 2. Fotografías de: A) Tamandua mexicana (IBUNAM-CFB-17410), tomada en bosque de pino-encino en la localidad de Concepción Pápalo, Oaxaca. B) Eira barbara (IBUNAM-CFB-17171), tomada en bosque mesófilo de montaña en la localidad de Santa María Pápalo, Oaxaca. C) Galictis vittata (IBUNAM-CFB-174), tomada en bosque mesófilo de montaña en la localidad de Santa María Pápalo, Oaxaca.

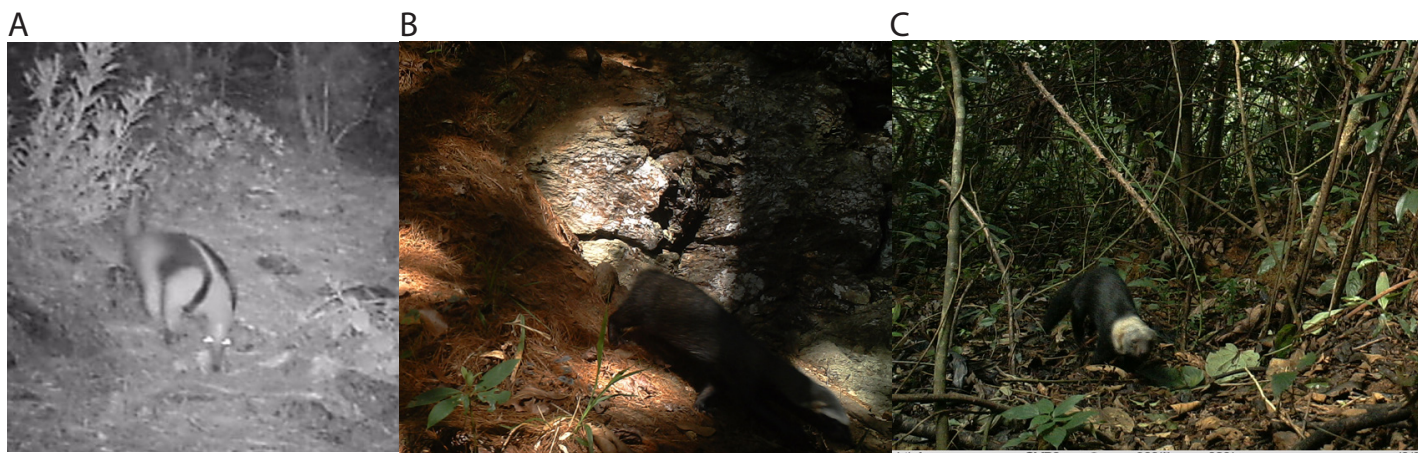


estas zonas del sureste de Oaxaca, aledañas a la Reserva, sean un punto importante en el flujo de especies entre la Chinantla y la RBTC. Con este escenario, es de resaltar la importancia del monitoreo participativo, que permite generar tanto el arraigo entre los comuneros como información científica básica. Asimismo, no debe perderse de vista que este esquema de investigación es una herramienta que potencialmente puede generar, a largo plazo, información robusta y estrategias de conservación en pequeñas y grandes escalas (Dickinson et al. 2010; Erb et al. 2012).

\section{Agradecimientos}

Se agradece a las autoridades comunales y a los comités de monitoreo participativo de Concepción y Santa María Pápalo. AE-L agradece al Consejo Nacional de Ciencia y Tecnología (CONACyT) y al Posgrado en Ciencias Biológicas de la UNAM (CVU: 479749).

\section{Literatura citada}

Aranda, J. M. 2012. Manual para el rastreo de mamíferos silvestres de México. Comisión Nacional para el Conocimiento y Uso de la Biodiversidad. Ciudad de México, México.

Bornholdt, R., K. Helgen, K. P. Koepfli, L. Oliveira, M. Lucherini, y E. EiziriK. 2013. Taxonomic revision of the genus Galictis (Carnivora: Mustelidae): species delimitation, morphological diagnosis, and refined mapping of geographical distribution. Zoological Journal of the Linnean Society 167:449-472.

Botello, F., E. Villaseñor, L. Guevara, A. Méndez, A. Cortés, J. Iglesias, M. Izúcar, M. Luna, A. Martínez, y J. M. Salazar. 2013. Registros notables del zorrillo manchado (Spilogale angustifrons) y del jaguarundi (Puma yagouaroundi) en la Reserva de la Biosfera de Tehuacán-Cuicatlán, Oaxaca, México. Revista Mexicana de Biodiversidad 84:713-717.

Briones-Salas, M. 2000. Lista anotada de los mamíferos de la Región de la Cañada, en el Valle de Tehuacán-Cuicatlán, Oaxaca, México. Acta Zoológica Mexicana (n. s.) 81:83103.

Ceballos, G., y G. Oliva (EDS.). 2005. Los mamíferos silvestres de México, primera edición. Comisión Nacional para el Conocimiento y Uso de la Biodiversidad, Fondo de Cultura Económica. Ciudad de México, México.

Cuarón, A. D. 2005. Tamandua mexicana. (Saussure, 1860) Oso hormiguero. Pp. 121-123 en Los mamíferos silvestres de México (Ceballos, G., y G. Oliva, eds.). Comisión Nacional para el Conocimiento y Uso de la Biodiversidad, Fondo de Cultura Económica. Ciudad de México, México.

Dávila, P., M. C. Arizmendi, A. Valiente-Banuet, J. L. Villaseñor, A. Casas, y R. Lira. 2002. Biological diversity in the Tehuacán-Cuicatlán Valley, Mexico. Biodiversity and Conservation 11:421-442.

Dickinson, J. L., B. Zuckerberg, y D. N. Bonter. 2010. Citizen science as an ecological research tool: Challenges and benefits. Annual Review of Ecology, Evolution, and Systematics 41:149-172.

Erb P. L., W. J. McSheay, y R. P. Guralnick. 2012. Anthropogenic Influences on Macro-Level Mammal Occupancy in the AppalachianTrail Corridor. PLoSONE 7:e42574doi:10.1371/ journal.pone.0042574

López, C. A., Y D. R. Aceves. 2007. Noteworthy record of the Tayra (Carnivora: Mustelidae: Eira Barbara) in the Sierra Gorda Biosphere Reserve, Querétaro, México. Western North American Naturalist 67:150-151.

Llorente-Bousquets, J., y S. Ocegueda. 2008. Conocimiento actual de la biodiversidad. Pp. 
283-322 en Capital Natural de México, Volumen 1 (J. Sarukhán, ed.). Comisión Nacional para el Conocimiento y Uso de la Biodiversidad. Ciudad de México, México.

Navarrete, D., y J. Ortega. 2011. Tamandua mexicana (Pilosa: Myrmecophagidae). Mammalian Species 43:56-63.

Nuñez-Perez, R., E. Corona-Corona, J. Torres-Villanueva, C. Anguiano-Méndez, M. Tornez, I. Solorio, y A. Torres. 2011. Nuevos Registros del Oso Hormiguero, Tamandua mexicana, en el Occidente de México. Edentata 12:58-62.

O' Connell, A., J. D. Nichols, y K. U. Karanth. 2011. Introduction. Pp. 1-8 en Camera Traps in Animal Ecology Methods and Analyses (O'Connell, A. F., J. D. Nichols, y K. U. Karanth, eds.). Springer. New York, EE. UU.

Oliveira, T. G. 2009. Notes on the distribution, status, and research priorities of little-known small carnivores in Brazil. Small Carnivore Conservation 41:22-24.

Pérez-Irineo, G., y A. Santos-Moreno. 2012. Diversidad de mamíferos terrestres de talla grande y media de una selva subcaducifolia del noreste de Oaxaca, México. Revista Mexicana de Biodiversidad 83:164-169.

Presley, S. J. 2000. Eira barbara. Mammalian Species 636:1-6.

Portal de datos de GBIF, www.GBif.net. 25-09-2015. Colección de Mamíferos del Museo de Zoología 'Alfonso L. Herrera'. México, Número de registro 5639.

Portal de datos de GBIF, www.gBif.net. 25-09-2015. The Museum of Comparative Zoology Collections, EE. UU. Número de registro 6424.

Ramírez-Bravo, O. E. 2011. Nuevos registros de tayra (Eira barbara Linnaeus 1758) en Puebla, centro de México. Acta Zoológica Mexicana (n. s.) 27: 883-886.

Ramírez-Pulido, J., N. González-Ruiz, y H. H. Genoways. 2005. Carnivores from the Mexican state of Puebla: distribution, taxonomy and conservation. Mastozoología Neotropical 12:37-52.

Ramírez-Pulido, J., y J. Martínez-Vázquez. 2007. Diversidad de los mamíferos de la Reserva de la Biosfera Tehuacán-Cuicatlán, Puebla-Oaxaca, México. Informe final Snib-Conabio proyecto No. BK022. Ciudad de México, México.

SEMARNAT. 2010. Norma Oficial Mexicana NOM-059- SEMARNAT-2010, Protección ambiental - Especies nativas de México de flora y fauna silvestres - Categorías de riesgo y especificaciones para su inclusión, exclusión o cambio - Lista de especies en riesgo. Diario Oficial de la Federación. 30 de diciembre de 2010, Segunda Sección. Ciudad de México, México.

SEMARNAT. 2013. Programa de Manejo Reserva de la Biosfera Tehuacán-Cuicatlán. Secretaria de Medio Ambiente y Recursos Naturales. Comisión Nacional de Áreas Naturales Protegidas. Ciudad de México, México.

Summited: August 18, 2015

Review: August 27, 2015

Accepted: September 10, 2015

Associated: Sergio Ticul Alvarez Castañeda 